\section{Cahiers de Narratologie}

Analyse et théorie narratives

12 | 2005

Récit et éthique

\title{
L'éthique dans les récits de Panaït Istrati
}

\section{Frédérica Zephir}

\section{OpenEdition}

Journals

Édition électronique

URL : http://journals.openedition.org/narratologie/223

DOI : 10.4000/narratologie.223

ISSN : 1765-307X

Éditeur

LIRCES

\section{Référence électronique}

Frédérica Zephir, «L'éthique dans les récits de Panaït Istrati », Cahiers de Narratologie [En ligne],

12 | 2005, mis en ligne le 20 avril 2005, consulté le 20 avril 2019. URL : http://journals.openedition.org/ narratologie/223 ; DOI : 10.4000/narratologie.223

Ce document a été généré automatiquement le 20 avril 2019.

\section{(c) $($ ) $(9)$}

Cahiers de Narratologie - Analyse et théorie narratives est mis à disposition selon les termes de la licence Creative Commons Attribution - Pas d'Utilisation Commerciale - Pas de Modification 4.0

International. 


\title{
L'éthique dans les récits de Panaït Istrati
}

\author{
Frédérica Zephir
}

1 Très heureuse de participer à ce cycle de conférences du C.N.A. grâce à la bienveillance de son directeur Monsieur Jean-Louis Brau, je suis d'autant plus contente que, grâce à son thème, il m'est permis d'aborder l'œuvre d'un écrivain qui, par les sujets qu'elle développe et par sa forme même, semble particulièrement apte à illustrer la réflexion sur

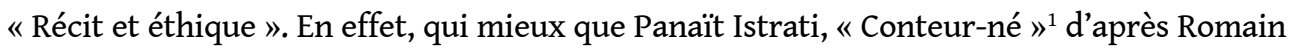
Rolland et «Pèlerin du cœur ${ }^{2}$, c'est-à-dire homme juste et généreux, selon Joseph Kessel, pourrait plus parfaitement incarner ces deux notions?

2 Panaït Istrati est né en 1884 à Braïla en Valachie. Fils d'une paysanne roumaine et d'un contrebandier grec, il a mené pendant vingt années une vie errante, parcourant la plupart des pays du bassin méditerranéen. Passionné, instable, il exerça, pour vivre, mille petits métiers et c'est au gré des occasions qu'il découvrit la littérature, notamment avec les grands romanciers russes. Epris de liberté et de justice, il participa un temps au mouvement socialiste qui s'éveilla en Roumanie à la veille de la première guerre mondiale.

3 C'est en 1920 qu'il arriva en France après un séjour de deux années en Suisse durant lequel il apprit le français et découvrit l'œuvre de Romain Rolland, qui fut pour lui une véritable révélation. Venu sur la Côte d'Azur pour y trouver du travail, il mène à Nice une existence quasi misérable. Seul, sans travail, sans argent, découragé, il se tranche la gorge le 3 janvier 1921 au jardin Albert $\mathrm{I}^{\mathrm{er}}$ au pied du monument de la Victoire. Transporté à l'hôpital Saint Roch, on découvre dans une de ses poches une longue lettre adressée à Romain Rolland et, dans ses papiers, un manuscrit de vingt-sept pages pour le même destinataire. Rolland ayant reçu ces deux envois, répond à Istrati et s'établit dès lors entre les deux hommes une relation épistolaire qui durera plusieurs années.

4 Frappé par la force qui se dégage de ces lettres, ayant vu (je cite) « luire, en éclairs, le feu divin de l'âme ", Rolland va pousser Istrati, lui donner confiance, le motiver, mais aussi l'exhorter à canaliser la flamme qui le dévore afin de l'amener à réaliser l'œuvre qu'il 
porte en lui «je n'attends pas de vous des lettres exaltées; j'attends de vous, des œuvres $»^{3}$ lui écrit-il en 1922. Ainsi, soutenu, guidé mais aussi, pour ainsi dire, sommé, par cette autorité littéraire incontestée, Istrati publie en 1924 Kyra Kyralina, la première des quatre œuvres formant le cycle des Récits d'Adrien Zograffi. Le succès est immédiat, Istrati fait une entrée fulgurante sur la scène des lettres françaises. Car c'est en français que ce photographe-ambulant roumain autodidacte livre, à quarante ans, une œuvre unanimement saluée par la critique de l'époque qui voit en lui (je cite) « un Gorki des Balkans ». Après ces premiers récits, Istrati en publiera plusieurs autres - dont Les Chardons du Baragan en 1928 présenté souvent comme son chef-d'œuvre.

5 Mais après le compte-rendu du voyage qu'il fit en U.R.S.S. entre octobre 1927 et février 1929, intitulé Vers l'autre flamme après seize mois dans l'U.R.S.S., sa fortune littéraire se retourna et c'est honni et calomnié par la presse de gauche qu'il mourut en 1935 à Bucarest.

6 Si ces récits, non seulement Kyra Kyralina, mais Oncle Anghel, Présentation des Haïdoucs et Domnitza de Snagov qui forment donc les Récits d'Adrien Zograffi ont connu un tel succès, c'est qu'ils apportaient un ton nouveau dans la littérature française de l'époque. Puisés aux sources de la mémoire de l'auteur, réceptacles de ses souvenirs d'enfance dans la cosmopolite cité de Brailla et de ses errances en Méditerranée, les récits d'Istrati s'abreuvent aussi aux origines de la tradition populaire roumaine. Si Kyra Kyralina baigne plus spécifiquement dans une atmosphère de conte oriental avec l'évocation de ses harems, de ses odalisques, de ses potentats turcs, de ses narguilés parfumés et de l'agitation chamarrée des villes de l'Orient, les autres récits font plus largement appel aux vieux fonds de la littérature orale commun à toutes les régions du Sud du Danube, et présent en Roumanie au moins depuis le XV ème siècle. C'est en effet les «cîntec haiducesc », les chants haïdouks, auxquels le poète roumain Valise Alecsandri (1821-1890) donna ses lettres de noblesse sous le nom de "ballades ", qu'évoque Istrati dans Cosma, troisième partie d'Oncle Anghel, Présentation des Haïdoucs et Domnitza de Snagov.

7 Se présentant sous la forme de récits enchâssés, la narration ressuscite les figures de haïdoucs, personnages historiques idéalisés en héros par la légende et la poésie épique orale. D'après Monique Jutrin-Klener, qui a consacré une thèse à l'œuvre de Panaït Istrati, et que je cite : «le mot haïdouc d'origine magyare apparaît en roumain au XVI ${ }^{\text {ème }}$ siècle, et prend à la fin du XVII ${ }^{\mathrm{ème}}$ le sens qu'on lui connaît dans les ballades populaires de horsla-loi révolté contre l'ordre social imposé par l'oppresseur turc $»^{4}$.

8 Et c'est bien en se fondant sur cette conception qu'Istrati va développer, non pas de façon didactique ou idéologique, mais véritablement en conteur oriental, sa vision du monde en exposant les valeurs qui sont les siennes; valeurs d'un passionné qui place la liberté, la justice, la bonté au-dessus de toute autre considération. Cependant, cette éthique de la générosité n'apparaît pas -tant s'en faut- comme une mièvrerie sentimentale et revêt au contraire un aspect fort complexe. D'abord parce qu'elle révèle, dans l'ensemble des quatre récits, une évolution qui la conduit de la simple révolte primitive de justiciers, certes vaillants et fraternels mais néanmoins violents et cupides, vers une lutte sociale organisée et un combat patriotique en prise directe sur l'histoire roumaine du milieu du $\mathrm{XIX}^{\text {ème }}$ siècle. Ensuite, et surtout, parce qu'elle débouche sur la conception extrêmement originale -pour ne pas dire unique pour l'époque- d'une éthique fondée à la fois sur la lucidité, la désillusion mais aussi sur une indéfectible foi en l'être humain et en la vie, qu'Istrati synthétisera dans la formule lapidaire : «l'homme qui n'adhère à rien » 
9 C'est ce double enjeu que nous voudrions maintenant mettre en lumière en exposant successivement trois conceptions de l'éthique telle qu'elle apparait dans l'œuvre, d'abord celle du pouvoir en place, à laquelle s'oppose l'éthique de la marginalité, et l'évolution de celle-ci au fil des récits, et enfin, l'éthique de la compassion qui paraît constituer l'ultime message qu'Istrati ait voulu livrer au terme de son œuvre.

Toute l'œuvre d'Istrati, ainsi que les valeurs qui s'en dégagent, se constitue en fonction du contexte historico-sociologique établi dans les principautés danubiennes -Moldavie et Valachie- à partir de la conquête ottomane du XIV ème siècle, et plus précisément à partir de la fin du XVII ème siècle marquée par la dégradation des conditions de vie des populations soumises.

11 Cet immense empire, obéissant aux ordres du Sultan de Constantinople, reposait en effet, dès le commencement, sur le principe intangible de toute conquête à savoir la division des sujets en deux catégories d'emblée inégales en droit : les vainqueurs et les vaincus; division qui, en l'occurrence, s'en doublait d'une autre non moins impérieuse, puisque les vainqueurs étaient musulmans et les vaincus chrétiens. A partir de cette organisation fondée sur la discrimination et l'inégalité, toutes les valeurs admises de gré -et le plus souvent de force- sont aux yeux, de la morale, des contre-valeurs et l'éthique du pouvoir en place, une contre éthique. Ainsi entre les sujets ottomans et les "raïas", sujets chrétiens toujours méprisés, entre les esclaves tziganes et les hommes libres, entre les grands féodaux, qui essayaient de sauver leurs intérêts en collaborant avec les conquérants, et les paysans spoliés de leur terre, l'injustice touchait toutes les strates de la société. La dénonciation de cette iniquité généralisée, de ce principe immoral et inhumain établi comme fondement de la société, est un des motifs récurrents de l'œuvre. $\mathrm{Au}$ travers des portraits des différents représentants du pouvoir, comme au travers de l'analyse de la condition sociale des paysans, Istrati s'élève puissamment contre cette injustice, à propos de laquelle il fait dire par exemple à l'un de ses personnages (je cite) :

« De tout temps, mes deux yeux ne voyaient autre chose que les forts trébuchant dans l'opulence, et les faibles tordus sous la cravache $»^{6}$.

Plus tard, Floarea Codrilor, l'héroïne de Domnitza de Snagov déclarera encore :

«A entendre le monde, Dieu voulait qu'il y eut des serfs et des gospodars, des pauvres et des riches, des fouettés et des fouetteurs $»^{7}$.

Dans cette évocation des figures du pouvoir, les portraits les plus saisissants sont ceux qui présentent les despotes locaux et leurs alliés, boïars secondés par les logofats, les représentants du clergé, et notamment les moines, enfin les agents du pouvoir étranger, auquel il faut ajouter le complice de tous les crimes, l'exécuteur des basses œuvres qu'est le « potérache », mercenaire sans foi ni loi enrôlé dans la sauvage " potéra ».

Le lourd ressentiment envers le boïar que révèle la quasi-totalité des récits, multipliant les exemples d'exactions et d'inhumanité, s'explique par le rôle que ce personnage a exercé durant l'occupation turque. Car c'est l'évolution historique qui a fait du boïar (du latin «bellum ») -titre au moyen-âge non héréditaire et réservé aux soldats- un véritable seigneur féodal. S'étant considérablement enrichis au cours des siècles, les boïars se sont en effet sentis particulièrement menacés par la domination ottomane et, durant les trois premiers quarts du XIX ${ }^{\text {ème }}$ siècle, ont fait subir une écrasante pression au petit peuple des campagnes. Le Divan de la Sublime Porte offrant les trônes conquis au plus offrant, les boïars durcirent encore leur emprise, confisquant les terres, pressurant le paysan en l'accablant sous toute sorte d'impôts ; je cite un passage de Domnitza de Snagov : 
«Les boïars se sont multipliés comme la mauvaise herbe, sont devenus injustes, rapaces et désireux chacun de régner ne fût-ce que quelques mois. Le trône étant [... ] aux mains des turcs, et se vendant au plus offrant, les nouveaux boïars eurent besoin d'argent pour se faire des partisans puissants dans le pays, et, à Stamboul, acheter les favoris du sultan. De là, vols et pillages. Ils n'avaient plus besoin de razechi, (c'est-à-dire de petits propriétaires) mais de beaucoup de terre, et pour la travailler, de serfs ${ }^{8}$.

15 Ainsi les principautés se couvrirent de nombreux latifundia gagnés en rongeant sournoisement les terres du paysan, sur lesquels régnait en maître absolu, le gospodar c'est-à-dire le boïar.

« Homme injuste, cruel et avide $»^{9}$ (je cite) le boïar considérait ses paysans comme des esclaves -en plus des véritables serfs qu'étaient les tziganes attachés à sa terre et vendus avec elle- ayant sur eux droit de vie et de mort, leur distribuant les parcelles de terre seule ressource pour vivre- selon son bon plaisir.

Ce seigneur cruel, "cette brute qui maîtrise la terre » exerçait aussi son pouvoir sur toutes les femmes et les jeunes filles de ses domaines qu'il considérait lui appartenir comme lui appartenait tout le reste. Les récits abondent en exemples de ces humiliations que subissaient les femmes, et que devaient accepter les hommes sous peine des représailles les plus brutales, allant du châtiment corporel à la mort, en passant par la privation des parcelles à cultiver. C'est ainsi que Floarea Codrilor, quand elle n'est encore qu'une jeune bergère, se voit poursuivie par le fils du boïar jusqu'à ce que Groza, son ami d'enfance, la délivre en neutralisant son agresseur, accomplissant alors son premier acte de révolte et signant par là son engagement dans le camp des haïdoucs; elle déclare :

«J'avais maintenant près de dix-sept ans. Et belle comme vous le voyez, cette beauté m'attira entre autres assiduités celle du fils de notre gospodar Bolnavul [...]. Il était quelqu'un, moi, j'étais quelque chose qui se tenait debout par hasard et devait s'allonger au premier signe du maître $»^{10}$.

18 Si le paysan, le « cojane », était assujetti corps et bien à la domination du boïar, il devait en outre subir la tyrannie des subalternes dont celui-ci s'entourait pour gérer ses domaines. Ainsi le « logofat », intendant aux pouvoirs discrétionnaires, prenant exemple sur son maître et répercutant à son niveau l'injustice qui régnait au sommet de la hiérarchie, rançonnait les paysans, les brutalisait, pouvait même les tuer, et considérait, lui aussi, les jeunes villageoises comme des sujets dont il pouvait disposer à sa guise. Le récit de Spilca dans Présentation des Haïdoucs fait apparaître ce crime comme une pratique entrée dans les mœurs, et pour Sultana, la jeune paysanne qui en est la victime, comme une véritable fatalité, le logofat devenant une incarnation de sa destinée. Remarquée par lui, elle ne peut plus décider librement de sa vie et se marier, car elle ferait inéluctablement le malheur de son époux. Considérée dès lors comme une "pacoste ", c'est-à-dire une calamité, par l'ensemble de la communauté villageoise, elle sait que rien ne pourra détourner son destin et qu'elle doit l'accepter, (je cite) :

«C'est le logofat Costaki [...] tu as peut être entendu parler de sa cruauté, de ses méfaits. Nous dépendons de lui comme tous les habitants : il peut nous laisser vivre ou nous tuer à sa guise. Et la jeune fille qui attire son attention ne peut pas lui échapper. Elle a le choix entre son déshonneur et la ruine de sa famille [...]. Cet homme n'a ni cœur ni honte. Il est notre maître [...] personne n'ose affronter le tyran $»^{11}$.

19 Tyranniques, cupides et cruels, les boïars versaient aussi parfois dans la franche débauche où ils se retrouvaient alors avec des membres du clergé, autres figures du pouvoir, dont les récits d'Istrati dévoilent sans détours l'hypocrisie, l'inhumanité et jusqu'à l'abjection. 
20 Bien que l'ensemble des récits offre des portraits d'ecclésiastiques parfaitement désintéressés et dévoués à la cause des humbles, à l'instar du prêtre du village martyr évoqué dans le récit de Movila, ou le chantre Joakime, qui abandonne son église et sa position privilégiée pour embrasser la cause des haïdoucs et s'y sacrifier, la plupart des représentants du clergé apparaissent cependant comme des rapaces assoiffés de richesses, dénués de toute morale et de toute humanité lorsqu'ils ne s'avèrent pas être de véritables monstres, pervertis et viciés jusqu'au tréfonds de leur être. Oublieux des préceptes chrétiens de pauvreté, le clergé spoliait en effet les biens des paysans et, à l'exemple du boïar dont il est perçu comme le complice, le pope les asservissait lui aussi à de nombreuses corvées (je cite) :

«Les hommes d'église [...] asservissaient le paysan au point de le faire travailler gratuitement la moitié de l'année $»^{12}$.

21 En conséquence de quoi, à l'instar des grandes propriétés seigneuriales, les institutions religieuses -églises et surtout monastères- prospéraient, étalant une richesse arrogante en regard de la misère qui régnait dans les campagnes. A ce propos, un des personnages de Domnitza de Snagov déclare au sujet du Supérieur du monastère Orbou :

«Son monastère est un de ceux qui ont raflé le plus de terre et de montagnes boisées aux pauvres communautés paysannes d'autrefois. Les esclaves tziganes fourmillent sur ses domaines $»^{13}$.

Outre les préceptes de pauvreté, les religieux bafouaient encore les principes de la charité chrétienne et, plus largement, de la plus élémentaire humanité, en pratiquant donc l'esclavage sur leur propriété, rabaissant les serfs au niveau de véritables bêtes de somme quand ils ne les enchaînaient pas comme des animaux. Domnitza de Snagov offre de cette condition une peinture saisissante au travers de l'évocation d'un des tziganes esclave du même monastère :

« Ces fers et cet usage [...] n'étaient ni plus ni moins qu'une barbarie : le tzigane qui se tenait devant nous [...] n'avait plus rien d'humain $»^{14}$.

Ce monastère apparaît d'ailleurs comme archétypique de la corruption régnant dans l'ensemble du clergé, cristallisant sur lui tous les vices qui, aux yeux d'Istrati, font de l'institution toute entière un instrument de tyrannie au même titre que le pouvoir seigneurial et le pouvoir étranger. Il n'est dès lors pas surprenant de trouver associés dans la plus ignoble abjection, tel que l'expose le récit d'Elie dans Présentation des Haïdoucs, les représentants de ces pouvoirs despotiques, unis grâce à leur force, pour ériger en norme de vie et de gouvernement des contre-valeurs morales dictées par l'égoïsme et le mépris de la personne humaine.

Ainsi asservi et humilié par les pouvoirs locaux du boïar et du clergé, le paysan devait encore subir les exactions commises par les hordes étrangères qui déferlaient à intervalles plus ou moins réguliers sur les Principautés. D'abord les turcs, avec les terribles janissaires envoyés par le Sultan en représailles du non-paiement du " haraciu » -le tribut dû par les peuples soumis. Pillant, violant, massacrant et brûlant les villages -à l'exception des biens du boïar qui avait acheté sa sécurité à prix d'or- cette soldatesque jetait sur les routes des convois de «béjénari », de fugitifs pitoyables, tel que les décrit le récit de Movila dans Présentation des Haïdoucs. Ensuite, les grecs, du patriote Ypsilanti et de son hétairie qui, partis en rébellion contre la domination turque en 1821, firent subir, malgré les assurances de leur chef, les pires déprédations aux populations autochtones, réitérant à leur encontre la même violence et les mêmes méthodes qu'ils étaient censés combattre. 
toute façon, la pression était omniprésente dans la vie du paysan et, qu'il s'agisse des Phanariotes grecs, des agas, pachas ou hospodars turcs, associés aux oppresseurs locaux, tous étaient unis pour exploiter la paysannerie. Je cite Istrati dans Domnitza de Snagov :

«... alors que les faibles se divisaient par nation et par religion pour maudire le mal les forts -turcs, grecs ou roumains- vivaient en harmonie et écrasaient sans distinction $»^{15}$.

Et pour protéger ses intérêts et combattre ceux en qui s'élevait un désir de révolte devant tant d'injustice et de misère, le pouvoir s'était doté d'un instrument à son image, aussi brutal, aussi inhumain, aussi corrompu que lui : la potéra, cohorte de bandits stipendiés par le boïar, qu'Istrati présente ainsi :

«Le Cârc-Serdar (c'est-à-dire le capitaine) partait avec sa potéra composée de deux cents mercenaires poursuivre les haïdoucs [...] fort heureuse de ne pas les rencontrer, ces sauterelles s'abattaient sur les villages, pillaient, violaient, torturaient, jetaient dans le désespoir un tas de communes innocentes $»^{16}$.

Parcourant ainsi le pays, et ajoutant ses méfaits à tous les autres, celle-ci achevait de faire de la condition paysanne des régions Moldo-Valaques une des plus misérables, socialement et bien plus encore humainement, d'Europe, à l'écart des idées de justice et d'égalité depuis longtemps déjà répandues par le Déclaration des Droits de l'Homme, et loin encore des mouvements révolutionnaires qui se répandaient peu à peu en Occident.

28 Ainsi, conteur dans l'âme, c'est au travers d'une narration alimentée aux sources de sa mémoire -souvenir de récits glanés au hasard de ses rencontres -qu'Adrien Zograffi, "double diégétique de Panaït Istrati $»^{17}$ - selon la formule d'Elisabeth Geblesco, livre dans ses récits, marqués par l'atmosphère orientale ou répercutant les échos de la tradition populaire, la peinture de la condition paysanne dans les principautés danubiennes. Condition dramatique résultant de l'exercice d'un pouvoir assis sur des valeurs perverties, sur une contre-éthique fondée sur l'égoïsme des forts et le mépris des faibles. Et c'est de la même façon que le récit, puisant dans le vieux fonds épique, va opposer à ces principes dépravés, les vraies valeurs de liberté, de justice et de respect de la personne humaine incarnées par les figures légendaires des haïdoucs. Définissant, au fil de la narration, les préceptes d'une véritable éthique de la marginalité, les multiples récits de Floarea Codrilor, de Jérémie, d'Elie, du mourrant de Bissoca -et d'autres encore- vont camper les puissantes personnalités de ces rebelles évoluant dans le "codrou », la forêt, espace privilégié de l'insoumission, et tracer enfin l'évolution qui les conduit d'une révolte primitive vers un idéal patriotique nettement défini.

Etymologiquement, la marge est ce qui s'éloigne du centre et donc, du point de vue axiologique, de la norme, c'est-à-dire de la règle comprise comme ce qui est imposé, et adopté, par tous comme ligne de conduite. Or, si les récits d'Istrati montrent bien comment les principes posés par le pouvoir sont unanimement acceptés par ses représentants dont il servent les intérêts, ils montrent aussi, comment ils sont, paradoxalement, admis par ceux-mêmes qui en sont les victimes. Tout le monde, en effet, accepte l'ordre établi aussi injuste et cruel soit-il. Par indifférence, par peur ou, plus insidieusement, parce qu'ils ont introjecté la norme, les opprimés se montrent rétifs à tout écart, à toute opposition et, les premiers, stigmatisent la rébellion contre la règle. Istrati, qui a très bien analysé ce mécanisme, écrit dans Présentation des haïdoucs :

«Cette tendre solidarité entre deux enfants qui se refusaient de baiser la main d'un pope complice du boïar, ainsi que d'ôter la caciula au passage de tout valet de la « cour » fut considérée comme un crime, non seulement par les intéressés, mais par ceux-là mêmes qui, étant serfs eux-mêmes, auraient dû suivre notre exemple $\aleph^{18}$.

Cahiers de Narratologie, 12 | 2005 
Or le haïdouc est justement celui qui, dès que sa conscience morale s'éveille, se distingue par sa façon de penser différente de celle des autres, celui qui n'accepte pas les choses telles qu'elles sont. Kyra, Spilca, Groza se sont ainsi tous démarqués de l'opinion commune en refusant -parfois dès leur enfance- le monde tel qu'il est institué par les lois injustes des hommes. Ainsi donc de Kyra, la sœur des futurs haïdoucs, Cosma et Elie, à propos de laquelle un des récits narre le fait suivant (je cite):

«Un autre jour, Elie prit une poignée d'or et la donna à un homme dont la maison venait de brûler avec bétail, outils et tout son avoir; Elie fut battu jusqu'au sang. Toute la maison approuva cette punition, sauf la petite sœur aux yeux de flamme. Elle fut battue à son tour pour avoir pensé autrement que la maison $»^{19}$. «Les haïdoucs qui étaient les seuls à ne pas penser comme tout le monde $»^{20}$ selon Floarea Codrilor sont alors très proches du rebelle, défini bien plus tard par Ernst Jünger dans son traité du même nom, c'est-à-dire de celui dont «les entreprises sont précédées d'une réflexion marquée par une conscience de l'imperfection des valeurs admises ${ }^{21}$.

Homme du refus, le haïdouc est aussi un sensitif, un passionné, mû par une empathie qui lui rend toute souffrance insupportable. "Homme-écho» chez lequel «tout résonne et qui aime à se mêler de tout ce qui est humain ${ }^{22}$ comme le définit Istrati, il ne peut rester insensible aux misères infligées à son prochain par l'iniquité des puissants, et se sépare donc de la société complaisante ou fataliste en s'engageant dans la voie de la révolte. Impitoyable envers les tyrans de toutes sortes comme envers les indifférents ou les résignés qui laissent faire, méprisant le danger et la mort même, le haïdouc dévoue sa vie à la cause de la justice. Libre dans sa pensée comme dans son existence, il se donne pour mission d'aider les opprimés, les faibles, en les vengeant des injustices et des humiliations subies de sorte que ses actes, quoique toujours orientés vers le bien, sont souvent marqués par la violence. Volant les riches pour secourir les pauvres, il lui arrive de tuer mais son crime n'est pas alors la conséquence du vice ou de sa cruauté, mais plutôt de celle de ses ennemis ou des circonstances. Animé par une sainte colère plutôt que par la haine, il agit en justicier et non en assassin car son idéal reste celui de la fraternité entre les êtres : «Haïr c'est bien, aimer c'est mieux. Seul celui qui sait haïr et qui peut aimer connaît la valeur toute entière de la vie $»^{23}$ dit Floarea Codrilor. Bandit de grand chemin, et malgré des débordements liés à la personnalité de certains individus, il reste néanmoins attaché à l'idée d'un brigandage idéal pur de tout égoïsme. Dès lors se dessine, dans l'œuvre d'Istrati, un premier aspect de l'éthique de la marginalité avec des haïdoucs émules de Robin Hood.

Organisés en petits groupes sous la houlette d'un capitaine au prestige incontesté, les haïdoucs, proscrits par la loi qu'ils récusaient et harcelés par les potéra, menaient une vie aventureuse et libre aux conditions très rudes. Brigands ou contrebandiers, ils ne sortaient des bois et des montagnes qui les abritaient que pour de foudroyantes incursions au cours desquelles ils châtiaient les boïars et leurs alliés, pillaient les monastères et secouraient, avec le fruit de leur rapine, les populations misérables. Instruisant Floarea Codrilor qui ignore encore leur existence, Groza lui décrit le haïdouc en ces termes :

«C'est l'homme qui ne supporte ni l'oppression ni les domestiques, vit dans la forêt, tue les gospodars cruels et protège le pauvre $»^{24}$.

Leur indéfectible dévouement à la cause des humbles, l'aide désintéressée qu'ils dispensaient sans ostentation, le réconfort moral que leurs actions apportaient en prouvant aux opprimés qu'ils n'étaient pas abandonnés à leur sort, et l'espoir que cela

Cahiers de Narratologie, 12 | 2005 
suscitait, faisaient des haïdoucs, à l'instar de Robin Hood et de ses compagnons, des héros aimés par le peuple qui les protégeait, admirait leur courage et chantait leurs exploits dans de longues ballades telle celle du haïdouc Gheorghitza qu'Istrati transcrit au premier chapitre de Domnitza de Snagov.

Cependant la frontière entre la pratique d'actes fondés sur la force et la violence -fussentils légitimés par la brutalité et la cruauté de l'adversaire- est mouvante et si ténue que les dérives étaient courantes dans les agissements des haïdoucs. Les récits, qui abondent en évocations de vengeances terribles et de châtiments impitoyables, mettent en effet en lumière le glissement qui menait parfois les justiciers d'actions nobles et généreuses vers des conduites viles et malhonnêtes, mettant ainsi à mal leurs principes moraux subvertis par des débordements condamnables. Elie, le frère de Cosma, reconnait ces dérapages en déclarant dans son récit qu'à une certaine époque ils furent, « des borfaches (c'est-à-dire des voleurs ordinaires) et que [leurs] vengeances furent [alors] mesquines et par trop intéressées $»^{25}$.

Souvent ces écarts reflétaient le tempérament du capitaine qui imprimait sa marque sur l'orientation générale des actions de la troupe. Chefs révérés, ces capitaines apparaissent dans les récits comme des personnalités exceptionnelles dont trois, Cosma, Groza et Foarea Codrilor, définissent le type du héros istratien.

Cosma, sans être le plus fidèle porte-parole de la pensée d'Istrati ni le meilleur représentant de l'éthique de la marginalité, est cependant le plus caractéristique des personnages, en ce sens qu'il représente le type même du haïdouc mis en scène dans l'œuvre ; Véritable Hercule des Carpates, il révèle déjà, par son physique impressionnant, sa parenté avec le héros de légende. De celui-ci il a la force, la vaillance et l'audace qui, le portant aux actions les plus périlleuses, lui confèrent une autorité incontestée parmi ses compagnons et une renommée prestigieuse dans le peuple. Mais par d'autres aspects de son caractère, il évoque aussi le héros moderne, romantique, tel que l'incarne par exemple Karl -personnage principal des Brigands de Schiller. Aveuglé par ses passions, il offre en effet une personnalité contrastée, à la fois "protecteur et tyran ${ }^{26}$ selon son frère, c'est-à-dire tour à tour bon et généreux, mais égoïste et dominateur; il est un personnage d'ombre et de lumière, capable du meilleur comme du pire. Poussé à la révolte par des motifs plus personnels qu'altruistes, il est devenu haïdouc pour défendre d'abord sa propre liberté à laquelle il est farouchement attaché. Mais il reste primaire dans sa conception de la vie selon laquelle l'individu doit avant tout satisfaire ses propres désirs et ne refreiner aucune de ses passions. Incapable de transcender cet égoïsme, il se laisse souvent emporter dans la voie de la déraison et accomplit alors des actes contraires à l'idéal moral des haïdoucs. Floarea Codrilor qui prendra sa succession à la tête de sa troupe, lui adresse ce reproche sévère :

«Tu ne connais pas la pitié. L'injustice ne te révolte que lorsqu'elle te touche toi seul, et pour satisfaire une de tes envies, tu broierais la terre ${ }^{27}$.

Personnage paroxystique dont les réactions, tant dans la douleur que dans la joie, sont marquées par l'excès, il porte en lui une violence qu'il dirige vers ses ennemis les boïars et les popes, puisqu'il est haïdouc, mais qu'il peut tout aussi retourner contre lui-même comme l'illustre sa réaction à la découverte de l'infidélité de Floarea Codrilor lorsque, submergé par la jalousie, il s'écrase la poitrine avec le sabot de son cheval.

De sorte qu'avec Cosma se dessine une figure de paladin à la charnière de la tradition et de la modernité. Empruntant à l'antiquité ses traits positifs, il est en effet le chef aux qualités exceptionnelles, la figure héroïque idéale, aimé du groupe qu'il conduit et aussi, à 
l'instar du héros classique, de la femme qu'il fascine et éblouit. Mais, par sa démesure, par cet abandon aux passions qui l'entraînent, Cosma est aussi, comme le proscrit Hernani, " une force qui va », c'est-à-dire un pur héros romantique que l'on a parfois justement défini comme un héros de la marginalité.

Mais au reste, Panaït Istrati qui, selon Monique Jutrin-Klener, aurait forgé Cosma à partir, de sa propre « joie de vivre, de sa fougue amoureuse [et] de sa jalousie féroce $»^{28}$ ne fut-il pas lui-même un être romantique?

41 A côté de ce personnage de violence et de passion en apparaît un autre qui se présente comme son opposé et dont l'importance réside non dans le rôle qu'il tient dans la diegèse, son existence de capitaine de haïdoucs n'étant pas raconté, mais dans sa conception de la morale, qui est celle mise en pratique par Floarea Codrilor dans Domnitza de Snagov et marque l'évolution de l'éthique de la marginalité.

Le personnage de Groza revêt en effet, du point de vue narratif, un statut particulier puisque le récit ne l'évoque qu'au début et à la fin de sa vie et encore de façon très elliptique, Istrati ayant d'abord envisagé de lui consacrer une œuvre entière, qu'il ne réalisa jamais et qui devait clore le cycle des Récits d'Adrien Zograffi. De sorte qu'il est difficile de saisir les raisons précises qui font de lui, dès l'enfance, l'incarnation des valeurs haïdouques idéales. Car dès l'âge de douze ans, ce garçon issu d'un hameau mal famé, possède en effet un sens moral particulièrement développé qui le différencie des jeunes, et même des adultes, qui l'entourent. Percevant en Floarea, la jeune bergère qu'il accoste un jour dans la prairie, une personnalité semblable à la sienne, il lui ouvre les yeux sur le monde et la sensibilise à la misère de la paysannerie. Refusant le servage auquel il est promis comme tous ceux de sa condition, il a déjà choisi sa destinée et sait qu'il sera très bientôt haïdouc ; se présentant à Floarea, il déclare d'emblée, « [Mon nom] est : Groza... Et je serai un jour haïdouc $»^{29}$. Devenu le « dascal », c'est-à-dire le maître, l'initiateur de la jeune fille, il va, "implanter dans (son âme) le germe de sa révolte innée $»^{30}$, en lui dévoilant les racines du mal dont souffre le paysan dépossédé de la terre par le boïar, l'éveillant à son tour à la conscience sociale. Dans le même temps, il la pousse à s'instruire et lui fait apprendre le grec auprès du chantre Joakime. Ayant châtié le fils du gospodar qui poursuivait Floarea de ses assiduités, et accompli ainsi son premier acte de haïdouc, Groza gagne la forêt et devient un capitaine redouté. C'est au moment de la séparation qu'il découvre à Floarea sa conception de la révolte, lui apprend dans quel but il l'a formée et ce qu'il attend d'elle, posant à cette occasion les principes de la nouvelle éthique haïdouque. Désormais, la lutte ne devra plus se borner à des actions certes généreuses mais limitées et sporadiques, mais prendre la forme d'une révolte organisée qui visera non plus seulement les seigneurs de moindre importance, "les petits méchants » comme dit un personnage, mais les grands boïars qui règnent à la tête des Principautés. «Il faut frapper haut ! $»^{31}$ dit Groza. Pour cela le haïdouc doit maintenant sortir des forêts et pratiquer la résistance de l'intérieur même du système, infiltrant, pour ainsi dire, les instances du pouvoir local et étranger. C'est cela que Groza attend de Floarea et c'est dans cet objectif qu'il l'a poussée à s'instruire. Je cite un extrait de l'ultime message qu'il lui adresse :

«En ville, parmi les gospodars, on peut être aussi bien haïdouc et révolté que l'homme qui vit «dans le cerveau des monts ", mais à condition d'être faux avec les grands et sincère avec les opprimés.

Tu sais être fausse et sincère : va donc, tâche d'aller au milieu des loups, hurle avec eux, observe leurs habitudes, connais bien leurs faiblesses et après, tire-leur dans le dos et fais du bien au peuple, venge-le [...]. 
Voilà, j'ai attendu ce jour pour te dire dans quel but je t'ai poussée à apprendre à lire et à écrire [...]. Il faut connaître le passé et le présent, pour savoir quoi désirer dans l'avenir. Travaille donc, pour cet avenir meilleur. On n'apprend pas le grec pour garder les brebis $»^{32}$. attestées en Bulgarie. historiquement attestés.
C'est sous l'influence de cette personnalité exceptionnelle que Floritchica, diminutif sous lequel apparaît Floarea Codrilor dans les premiers récits, va devenir capitaine de haïdoucs. Cette « Fleur des bois » comme l'indique son nom, prend en effet, dès avant sa mort, le commandement de la troupe que Cosma, consumé par la violence des passions, lui abandonne et devient à son tour un chef admiré et respecté. L'accession d'une femme à cette position traditionnellement occupée par des hommes trouve son origine, selon Monique Jutrin-Klener, dans le personnage de Voïca, héroïne d'un roman de Popescu intitulé Fata de la Cozia (sœur de la Croix) dont le modèle serait lui-même à rechercher dans la poésie bulgare, inspirée de figures de capitaines féminins historiquement

Après avoir connu et aimé Cosma, avoir eu de lui un fils, Jérémie qui deviendra lui-même haïdouc, Floritchica, mûe par une inextinguible soif de justice, et mettant en pratique les préceptes de son ami d'enfance, parcourt les contrées de l'empire ottoman revêtue, (je cite) « du masque de la fausseté $»^{33}$. Jouant le jeu des puissants pour mieux les combattre, servie en cela par sa grande beauté et son intelligence, c'est au bras de l'archonte Samourakis rencontré à Constantinople, qu'elle revient en pays roumain. Retrouvant à sa cour son fils esclave, elle assiste, sans toutefois y participer activement, à l'attaque du palais de l'archonte par Cosma avec lequel elle repart vivre dans la forêt. A partir de ce moment, prenant le commandement de la troupe à laquelle elle assigne une mission nouvelle, elle dévoile sa véritable personnalité désormais pleinement réalisée. Douée d'une force de caractère peu commune, riche de l'expérience forgée durant son immersion dans le monde, elle se révèle à la fois femme d'esprit et femme d'action. Réunissant en elle la volonté de Cosma et la raison d'Elie, elle apparaît, à un niveau d'analyse plus profond, comme la synthèse des personnalités opposées de Cosma et de Groza, c'est-à-dire des deux hommes auxquels elle s'est livrée de corps et d'âme.

Réalisant ainsi dans son être social son « rêve impossible » d'un amour à la fois fraternel et charnel, et harmonisant en sa personne de capitaine les contradictions de Cosma, elle incarne les valeurs idéales de l'éthique haïdouque. Et c'est donc bien, nous semble-t-il, la maturité morale et politique de Groza, plus que la sagesse exemplaire mais par trop détachée d'Elie, qui font de Floarea Codrilor la figure fascinante qui, devenue Domnitza de Snagov, participe de façon décisive à la lutte patriotique dans laquelle elle engage sa troupe de justiciers hors-la-loi.

Clôturant le cycle des Récits d'Adrien Zograffi, Domnitza de Snagov se présente comme une fiction en prise directe sur l'histoire. Couvrant une période de douze années de 1854 à 1866, elle évoque les grands événements historiques qui ont abouti à la création de la Roumanie moderne : la guerre de Crimée, l'intervention de la France et de l'Angleterre en faveur du futur état, l'accession au trône d'Alexandre Couza et les principales réformes faites sous son règne. C'est dans ce contexte référentiel que l'héroïne définit les principes de la nouvelle éthique des haïdoucs, développe une conception novatrice de la révolte, et engage ses compagnons dans une mission dont le but se confond avec les faits

Devenue Domnitza de Snagov, c'est-à-dire la Dame -au sens médiéval du terme- de Snagov, Floarea Codrilor s'éloigne de la révolte conçue sous la forme d'expéditions 
punitives telle que l'avait incarnée Cosma, pour promouvoir une action moins violente, inscrite à l'intérieur des cadres de la légalité. Cette "haïdoucie diplomatique ${ }^{34}$, telle qu'elle la qualifie, représente alors un bouleversement radical de la tradition puisque le haïdouc cesse d'être un hors-la-loi, s'intègre à la société, en respecte -au moins en apparence- les règles, et doit donc abandonner toute pratique illicite pour ne se consacrer qu'à l'amélioration du sort des humbles, (je cite) :

«[Floarea Codrilor] bouscula les bases de la haïdoucie primitive, en professant

d'emblée son mépris pour tout révolté qui n'était pas doublé d'un idéaliste $»^{35}$.

Abandonnant la forêt, le haïdouc va se fondre dans la population, devenant paysan ou exerçant un métier dans lequel, côtoyant les opprimés, il va employer toute son énergie à les éduquer, les conscientiser afin de les amener à s'organiser et à mener une lutte efficace contre le système qui les assujettit, (je cite) :

«On leur demandait maintenant de devenir de braves charretiers, d'éclairer tout un peuple abruti et de mettre des gants pour saccager un nid de vipères $»^{36}$.

49 De sorte qu'en pratiquant ainsi, les révoltés s'éloignent définitivement du modèle légendaire incarné par Robin Hood et se rapprochent d'une conception moderne de la résistance telle qu'elle fut illustrée, par exemple plus tard, par les dissidents des régimes totalitaires, très proche une nouvelle fois de la figure élaborée par Ernst Jünger dans son Traité du Rebelle qui affirme :

«Il existe des forêts au désert comme dans les villes où le rebelle vit caché sous le masque de quelque profession $»^{37}$.

50 Donnant la première l'exemple de cette mutation, en capitaine de haïdoucs qu'elle continue d'être, Floarea Codrilor se métamorphose en Domnitza, brillante maîtresse du domaine de Snagov qui accueille en hôtesse raffinée les élites réformatrices du pays, les ambassadeurs étrangers soutenant leur cause, et les représentants les plus conservateurs de l'aristocratie locale qu'elle tente de convertir aux idées libérales.

51 Si l'action des haïdoucs change ainsi de visage, c'est que les enjeux de la révolte se sont eux-mêmes transformés. Il ne s'agit plus en effet de mener des attaques vengeresses limitées et de dispenser quelque secours au paysan accablé, mais bien d'organiser une tentative de soulèvement afin d'éradiquer le système inégalitaire en redistribuant les terres, en abolissant l'esclavage des tziganes et la peine de mort, et en sécularisant les biens du clergé. Les haïdoucs se trouvent donc appelés à mener une véritable lutte sociale à laquelle vont s'associer -autre changement notoire- tous les membres de la société y compris les boïars réformistes sincères. Et la réalisation d'une telle alliance atteste bien alors l'évolution de l'éthique haïdouque tout comme elle définit celle d'Istrati lui-même, pour qui la générosité et le sens de la justice transcendent la notion de classe sociale, je cite Floarea Codrilor :

«Partager le monde en ceux d'en haut et en ceux d'en bas, n'attribuer aux premiers rien que des défauts, et aux seconds rien que des qualités et vouloir détruire les uns, pour livrer la terre aux autres, ce ne serait rien changer à la vie d'aujourd'hui [...].

Ce serait faire preuve d'une injustice criante que de dénier à un homme générosité, bonté, droiture, parce que sa naissance l'a placé parmi les étrangleurs $»^{38}$.

52 Ce ralliement de toutes les énergies favorables à une transformation de la société était d'autant plus nécessaire qu'il était impossible de ne compter que sur la volonté du peuple, insuffisamment éclairé, pour entreprendre un tel projet social, lequel se doublait de plus du projet politique d'union des Principautés en un état gouverné par un seul prince. C'est pourquoi, la fiction fait alors apparaitre Jiano, fils de grand boïar et compagnon des 
haïdoucs de Snagov, au côté de Miron et Couza, personnages historiques, premiers dirigeants de la Roumanie unifiée de 1859 à 1866.

Le dernier des ouvrages composant le cycle des Récits d'Adrien Zograffi marque donc, à l'évidence, une évolution de l'éthique de la marginalité. Avec Domnitza de Snagov, la morale des haïdoucs devient en effet plus pragmatique et leur action plus politique, sans pour cela se départir des valeurs de justice et de générosité qui en constituent l'essence. Car, en refusant, sous l'effet d'une lucidité quasi prophétique pour l'époque, le renversement de situation consistant à transférer simplement le pouvoir des oppresseurs aux opprimés, Istrati pose, comme précepte fondamental et comme but ultime de l'action, une véritable éthique de la générosité car dit Floarea «ce n'est pas l'homme tout court qui doit faire l'humanité, c'est l'homme bon $»^{39}$.

Parce qu'il est indissociable de la figure du haïdouc, révolté et proscrit, le "codrou », c'est-à-dire la forêt, ne peut être séparé de la notion de marginalité.

Tout d'abord lieu de refuge, elle est pour lui l'espace protecteur dans lequel il se replie pour échapper aux potéra lancées à sa poursuite. Retraite toujours ouverte dont il a une connaissance parfaite, il sait se retrouver dans ce milieu hostile pour tout autre que lui, qui y voit au contraire un lieu fraternel, régi par des lois naturelles bien moins injustes et cruelles que celles des hommes. Fraternel aussi parce que «le codrou, frère du Roumain ", selon un proverbe cité par Istrati, apparaît au haïdouc comme le reflet de l'humanité qui souffre mais garde toujours une lueur d'espoir. Généreuse, bienveillante envers les hommes auxquels elle offre ses présents, la forêt est en effet pour lui, (je cite) :

«La grande esclave qui vit pour créer le bonheur d'un monde ingrat [...] mais [qui] pendant que la hache la frappe à la racine [...] chante [à son faîte] ses derniers hymnes au soleil $»^{40}$.

Parfois encore vierge, la forêt sauvage, profonde, est le lieu d'élection de tout ce qui s'épanouit en dehors des cadres de la société et de ses règles. Terrain de liberté qui abrite et protège les brigands comme les rebelles, elle est l'espace privilégié de l'insoumission. Ainsi le codrou est-il largement représenté dans les récits d'Istrati, dans les ballades qu'il transcrit, comme dans l'évocation des lieux de repli des haïdoucs. Tazlau, les Bois de la Bâsca, le Bois du cerf, le Vallon obscur et la Grotte aux ours forment ainsi autant d'espace de la marginalité où se retrouvent les hors-la-loi pour s'abriter, préparer leurs prochaines incursions ou poser les bases de leur nouvelle mission. Et c'est enfin dans le codrou, insondable, incommensurable comme la mer et comme elle, symbole de liberté, que la fleur de la forêt, Floarea Codrilor exige de reposer, refusant tout autre lieu de sépulture, (je cite) :

«Et puis, j'ai toujours su que je finirais dans le codrou. Il n'y a que deux genres de tombes qui soient dignes d'enfermer les cœurs généreux : les océans et les fourrés » ${ }^{41}$.

Si la forêt, espace privilégié de la marginalité, est donc bien évoquée dans les récits, ceuxci en dévoilent un autre non moins caractéristique en ce qu'il reflète symboliquement l'évolution de l'éthique des haïdoucs. Grand domaine à l'image de ceux que possédaient les boïars, la maison de Snagov, précisément située aux abords d'un vaste codrou, et dissimulée, comme sa propriétaire, derrière des apparences respectables, se révèle en effet comme le lieu où se concrétisent leurs valeurs morales. Dépourvue de clôture, largement ouverte, sise au milieu du domaine duquel Floarea émancipa tous les esclaves tziganes dès son acquisition, la maison apparaît comme le centre réel et symbolique de la révolte, désormais poursuivie sous d'autres formes, mais toujours avec le même idéal, par 
les compagnons de Domnitza venus à Snagov "avec le désir de changer la face du monde $»^{42}$. C'est dans cette demeure de «l'amitié, de l'amour et des luttes généreuses $»^{43}$ où se construit le futur état roumain que les haïdoucs enthousiastes apprennent l'accession de Couza au trône de Moldavie, premier acte de l'unification des Principautés. Mais le règne de Couza fut bref et, malgré les réformes réalisées, le pays ne fut pas pour autant soustrait à l'injustice et rendu à la liberté. Aussi, assiégée par les "ciocoï », arrivistes plus féroces encore que les boïars et nouveaux maîtres du pays, la maison de Snagov finit-elle dans un gigantesque incendie qui efface jusqu'aux traces de l'idéal qu'elle représentait.

Et cette fin qui, dans le récit, annonce le déclin des haïdoucs, la mort de Floarea, la dispersion et l'errance de ses compagnons, traduit aussi le désenchantement dont l'auteur fait part dans sa préface. Revenu, après une absence de dix ans, dans ce qui était devenu la « Grande Roumanie », Istrati fut en effet bouleversé et indigné de constater que la liberté et la justice y étaient de nouveau bafouées. C'est sous le coup de cette désillusion qu'il renonça à écrire le dernier récit du cycle qu'il avait projeté.

59 A la marge de la société, le héros istratien, identifié au haïdouc, est donc celui qui, poussé par sa conscience et son amour de la liberté, décide de rompre avec la société et choisit, en ayant « recours aux forêts $»^{44}$ selon l'expression de Jünger, de partir en guerre contre l'injustice et la tyrannie. Version balkanique de Robin des Bois, les rebelles des récits, entrainés par trois capitaines d'exception, évoluent d'une révolte primitive encore proche des modèles épiques colportés par les ballades et les romans populaires, vers une lutte sociale et politique en prise directe sur le réel historique de la Roumanie de la fin du XIX ${ }^{\text {ème }}$ siècle.

Ce faisant, les Récits d'Adrien Zograffi définissent au fil de leur déroulement l'éthique, à la fois singulière et subversive, à laquelle aboutit Istrati à la fin de cette œuvre.

61 Car c'est bien une vision du monde, émergeant naturellement de la trame de la narration, qui se dégage des récits d'Istrati. Vision lucide et désabusée d'abord d'une humanité féroce mûe par l'égoïsme et l'intérêt, et d'un individu enfermé dans sa solitude par la profondeur et la singularité de ses émotions qui rendent toute expérience affective -dans la joie comme dans la douleur- incommunicable. C'est la leçon d'Oncle Anghel au terme d'une vie accablée par les malheurs, (je cite) :

« Tu as souffert, et tu souffres tes malheurs, mais pas les miens [...] Si tu perds demain ton fils, moi, je souffrirai, mais toi, tu mourras $»^{45}$.

Désillusion aussi face à une société dont la population opprimée est cependant réfractaire à tout bouleversement, ceux qui en émergent ne pensant qu'à reproduire à leur profit le modèle social injuste dont ils sont issus car, je cite Floarea Codrilor, «il est rare de trouver des étranglés qui n'aient jamais voulu être des étrangleurs $»^{46}$. Quant à la masse, inculte, passive et lâche, effrayée par l'audace des actions subversives elle n'offre aucun soutien au révolté, plus sensible au pouvoir du discours à «la magie des mots $»^{47}$ dit Istrati -du pope hypocrite ou du boïar despote- qu'aux actes généreux du rebelle. C'est contre elle aussi que doit lutter le haïdouc dont l'action n'est parfois récompensée que par l'incompréhension, quand ce n'est pas l'ingratitude. C'est la leçon de Groza répondant aux insultes d'une procession paysanne après la chute de Snagov et son arrestation par la potéra :

«Mais non! [...] Nous ne sommes pas des «bandits» ni des «assassins!». Nous sommes des haïdoucs! [...] Heureusement que je n'ai jamais compté sur votre appui, autrement, il y aurait de quoi s’écrabouiller la cervelle $! »^{48}$. 

changement et rendre vaine l'action même des justiciers mais auquel Istrati oppose justement les préceptes d'une morale et d'une action fondés sur la générosité. amélioration de la société par l'action de ceux qui, ayant maitrisé en eux la violence des instincts primaires par une élévation de cœur et d'âme, mettent leur révolte au service de la justice et de la liberté. «La bonté, unique distinction de la vie humaine » selon lui, peut sauver l'humanité et la révolte de l'homme généreux n'est donc jamais inutile. La maison de Snagov est détruite, les haïdoucs dispersés, Floarea, Elie, Groza disparus mais l'union des principautés a été réalisée, l'esclavage et la peine de mort abolis et Jérémie, le fils de Floarea, en compagnie de Bouzdougan, «iron[t] ensemble (je cite la dernière phrase de l'œuvre) répandre dans le monde le meilleur de [leur] jeunesse et de [leur] bonté $~_{49}$. «Un monde où tout finit, mais aussi tout commence par une chanson haïdouque $\|^{50} c^{\prime}$ est-àdire où le combat pour la justice et la liberté ne s'achève jamais. Ainsi se parachève la morale d'Istrati qui voit dans la lutte, et la résistance, un des moteur de la vie (je cite) :

«Combattre pour une idée, combattre pour un sentiment, pour une passion ou pour une folie, mais croire en quelque chose et combattre, voilà la vie. Qui ne sent pas la nécessité du combat, ne vit pas mais végète $»^{51}$.

Lutter contre la médiocrité humaine, l'égoïsme, l'injustice, être libre et défendre la liberté voilà donc la philosophie d'Istrati. Mais celle-ci, induite chez lui par la compassion et relevant donc plus du sentiment que des idées, ne trouva pas à se satisfaire dans les idéologies sociales de l'époque, trop doctrinales et totalitaires, qu'il dénonça et rejeta avec la même véhémence qu'il avait toujours mise à défendre les valeurs humaines fondamentales. De sorte que plus révolté, plus subversif, plus haïdouc que jamais, cristallisant son refus dans la figure de «l'homme qui n'adhère à rien» Istrati s'inscrit définitivement dans l'éthique de la marginalité.

« Homme qui n'adhère à rien » mais qui croit en l'homme, qui fait du combat pour les valeurs morales un des moteurs de la vie, et de la compassion un idéal social, mais surtout, surtout, conteur exceptionnel qui dévoile au fil de ses récits les histoires extraordinaires de ces héros -brigands souvent, révoltés passionnés et généreux, toujours- qui combattent au nom de la justice et de la liberté, tel fut Panaït Istrati « haïdouc de génie ».

\section{BIBLIOGRAPHIE}

Les citations d'Istrati sont extraites des éditions suivantes

Kyra Kyralina, Gallimard, collection Folio, 1968

Oncle Anghel, Gallimard, l'Etrangère, 1968

Présentation des Haïdoucs, Gallimard, collection Folio, 1968

Domnitza de Snagov, Gallimard, collection Folio, 1968 
Autres ouvrages consultés

ALBOUY, Pierre. Mythes et Mythologies dans la littérature française, Armand Colin, collection U, Lettres, Paris, 1998-2003

BRUNEL, Pierre. « Romantisme français et Romantisme européen » in Romantismes européens et Romantisme français sous la direction de P. Brunel, Institut Collégial européen, Editions Espaces 34,2000

Correspondance intégrale Panaït Istrati - Romain Rolland, 1919-1935, Etablie et annotée par Alexandre Talex, canevas Editeur, 1989

JORGA, Nicolas. Histoire des roumains et de leur civilisation, Henry Paulin Editeur, Paris, 1920

JÜNGER, Ernst. Le Traité du Rebelle ou le recours aux forêts, traduit de l'allemand par Henri Plard, Christian Bourgeois Editeur, Paris, 1981-1995

JUTRIN-KLENER, Monique. Panaït Istrati, Un chardon déraciné,François Maspero Editeur, Paris, 1970 Mythologie du Romantisme, La Licorne, 1990. Actes du colloque de Clermont-Ferrand 10 et 11 mars 1989 - Publication de l'U.F.R. de langues et littératures de l'Université de Poitiers 18

RAYDON, Edouard. Panaït Istrati, Vagabond de génie, Editions Municipales, 1968

SANTRAUD J.M., Geblesco E., Rossi C., Jutrin-Klener M., Popovici M., Lenz H., Lérault D., Les Haïdoucs dans l'œuvre de Panaït Istrati, L'insoumission des vaincus, Critiques littéraires, L'Harmattan, 2002

YOUKOV, Yordan. Légendes de la Stara Planina - Traduction de Roger Bernard, Editions en langues étrangères, Sofia, 1963

\section{NOTES}

1. Romain Rolland, Préface de Kyra Kyralina

2. Joseph Kessel, « Le Matin », 2 avril 1934

3. Panait Istrati - Romain Rolland, Correspondance, p. 90

4. M. Jutrin-Klener, Panaït Istrati, Un chardon déraciné, p. 176

5. P. Istrati, Préface à la maison Thüringer

6. Présentation des Haïdoucs, p. 85

7. Ibid, p.35

8. Domnitza de Snagov, p. 27

9. Présentation des Haïdoucs, p. 48

10. Présentation des Haïdoucs, p. 48

11. Ibid, p. 118

12. Présentation des Haïdoucs, p. 81

13. Domnitza de Snagov, p. 41

14. Ibid, p. 52

15. Présentation des Haïdoucs, p. 83

16. Ibid, p. 81

17. E. Geblesco, Présence Turque en Roumanie dans l'œuvre de Panaït Istrati, p. 59

18. Présentation des Haïdoucs, p. 32

19. Oncle Anghel, p. 121-122

20. Présentation des Haïdoucs, p. 35

21. E. Jünger, Traité du rebelle, p. 58 
22. Domnitza de Snagov, p. 80

23. Présentation des Haïdoucs, p. 58

24. Présentation des Haïdoucs, p. 58

25. Ibid, p. 93

26. Oncle Anghel, p. 114

27. Ibid, p. 193

28. M. Jutrin-Klener, op cité, p. 146

29. Présentation des Haïdoucs, p. 21

30. Ibid, p. 35

31. Ibid, p. 56

32. Présentation des Haïdoucs, p. 56-57

33. Présentation des Haïdoucs, p. 66

34. Domnitza de Snagov, p. 166

35. ibid, p. 65

36. Domnitza de Snagov, 10-49

37. E. Jünger, Op. Cit., p. 116

38. Domnitza de Snagov, p. 66-68

39. Ibid, p. 72

40. Présentation des Haïdoucs, p. 176-177

41. Domnitza de Snagov, p. 184

42. Ibid, p. 80

43. Ibid, p. 81

44. E. Jünger, Op. Cit

45. Oncle Anghel, p. 57-58

46. Domnitza de Snagov, p. 66

47. Ibid, p. 30

48. Ibid, p. 205

49. Domnitza de Snagov, p. 216

50. Ibid, p. 215

51. Panaït Istrati, Mes Départs in M. Jutrin-Klener, Op. Cit, p. 156

\section{AUTEUR}

\section{FRÉDÉRICA ZEPHIR}

Membre associé, CIRCLES 